\title{
mRNA expression of delta-HER2 and its clinicopathological correlation in HER2-overexpressing breast cancer
}

\author{
RYUICHI WADA $^{1,2}$, SOROKU YAGIHASHI ${ }^{2}$ and ZENYA NAITO ${ }^{1}$ \\ ${ }^{1}$ Department of Integrated Diagnostic Pathology, Nippon Medical School, Tokyo 113-8602; \\ ${ }^{2}$ Department of Pathology and Molecular Medicine, Hirosaki University Graduate School of Medicine, \\ Hirosaki, Aomori 036-8562, Japan
}

Received September 22, 2015; Accepted September 29, 2016

DOI: $10.3892 / \mathrm{mmr} .2016 .5892$

\begin{abstract}
Delta-human epidermal growth factor receptor 2 (HER2) is a splice variant of HER2, which lacks 16 amino acids in the extracellular domain. The aim of the present study was to elucidate the expression of delta-HER2 and its clinicopathological correlation in Japanese patients with HER2-overexpressing breast cancer. A total of 40 cases of HER2-overexpressing breast cancer were investigated. The mRNA expression levels of wild type (wt)-HER2 and delta-HER2 were quantitated by quantitative polymerase chain reaction using RNA extracted from formalin-fixed paraffin-embedded specimens. In addition, the activated form of phosphorylated-SRC (pSRC) and Ki-67 were detected by immunostaining. The mRNA expression levels of wt-HER2 varied largely, and the mRNA levels of delta-HER2 were correlated with those of wt-HER2. Furthermore, the enhanced immunostaining of pSRC was detected in 15 cases. Unsupervised hierarchal clustering of wt-HER2, delta-HER2 and PSRC was used to separate the cases into three clusters. In the first cluster, the levels of wt-HER2 and delta-HER2 were low, and pSRC immunostaining was low. Two other clusters were characterized by moderately and highly increased levels of wt-HER 2 and delta-HER 2 mRNA expression and enhanced $\mathrm{pSRC}$. The delta/wt ratio was higher in the first cluster. Positive lymph node status and recurrence were more frequent in the first cluster compared with those in the two other clusters. Furthermore, the delta/wt ratio was significantly increased in lymph node-positive and recurrent cases, compared with in lymph node-negative and non-recurrent cases. The present study demonstrated that delta-HER2 was expressed in Japanese patients with HER2-overexpressing breast cancer. High mRNA expression levels of delta-HER2 were associated with pSRC and increased proliferation of tumor cells. A poor
\end{abstract}

Correspondence to: Dr Ryuichi Wada, Department of Integrated Diagnostic Pathology, Nippon Medical School, 1-1-5 Sendagi, Bunkyo-ku, Tokyo 113-8602, Japan

E-mail:w-ryuichi@nms.ac.jp

Key words: HER2, splice variant, delta-HER2, Ki-67, breast cancer prognosis may be predicted by the increase in delta/wt ratio in HER2-overexpressing breast cancer.

\section{Introduction}

Human epidermal growth factor receptor 2 (HER2)-overexpressing breast cancer constitutes $20 \%$ of breast cancer cases (1). Overexpression of the HER2 protein is caused by amplification of the HER2 gene. HER2-overexpressing cancer often has a highly aggressive phenotype and is associated with metastasis to the lymph nodes and distant organs; among breast cancer types the prognosis for this type of cancer is the worst (2-4). Using anti-HER2 antibodies as molecular target-based therapy may ameliorate the prognosis of HER2-overexpressing breast cancer (5). However, the molecular mechanism underlying the aggressive behavior of HER2-overexpressing breast cancer remains to be fully elucidated.

Delta-HER2 is a splice variant of HER2, which lacks the 16 amino acids (coded by 48 nucleotides) of exon 16 in the extracellular domain (Fig. 1) (6,7). The deletion of these 16 amino acids in the cysteine-rich region generates unpaired cysteine residues, which cause chemical dimerization of the molecules and constitutive stimulation of cell proliferation $(8,9)$. It has been reported in vitro that overexpression of delta-HER 2 in cultured cells results in the generation of highly proliferative cells (8-12). Furthermore, in transgenic mice overexpressing delta-HER2, mammary carcinoma frequently developed, whereas overexpression of wild type (wt)-HER2 did not induce tumor formation (13). The findings of these in vitro experiments and animal models indicated that delta-HER2 is oncogenic.

The expression levels of delta-HER2 have been examined in cell lines and in human breast cancer tissues $(8,9,11,12,14)$; however, in some studies the number of patients is limited and the expression was examined by semi-quantitative methods $(9,14)$. The expression of delta-HER2 and its association with the clinicopathological factors of human breast cancer remain to be fully elucidated. Furthermore, to the best of our knowledge, the expression levels of delta-HER2 in Japanese patients with HER2-overexpressing breast cancer remain unknown.

The present study established a quantitative method to detect delta-HER2 mRNA expression in formalin-fixed paraffin-embedded cancer specimens. The aim of the 
present study was to explore the expression of delta-HER2 and its clinicopathological correlation in Japanese cases of HER2-overexpressing breast cancer.

\section{Patients and methods}

HER2-overexpressing breast cancer and histological evaluation. A total of 40 cases of breast cancer, which were given a score of 3 by HER2 immunostaining, were retrieved from the archival files. The present study was performed using formalin-fixed paraffin-embedded specimens of primary breast cancer in all cases except case \#8. In this case, the specimen was obtained from the metastatic lymph nodes, and information regarding the primary tumor was not obtained. The present study was approved by the Ethical Committee of Hirosaki University (Hirosaki, Japan). The histology of the breast cancers was classified according to the World Health Organization Classification of Breast Tumors (15). The HER2 immunostaining score was determined according to the guidelines recommended by American Society of Clinical Oncology/College of American Pathologists (16). No patients received preoperative neoadjuvant therapy.

Total RNA extraction and synthesis of cDNA. Total RNA was extracted from the paraffin-embedded sections using RNeasy FFPE kit (Qiagen K.K., Tokyo, Japan). Briefly, five slices from the $4 \mu \mathrm{M}$-thick paraffin-embedded sections were deparaffinized using xylene and ethanol, and dried. Subsequently, the sections were digested with proteinase $\mathrm{K}$ solution until the tissues were completely dissolved. Total RNA was purified using spin columns, according to the manufacturer's protocol. As a standardization control, cDNA reverse-transcribed from a mixture of total RNA extracted from the normal breast tissues of five women (age, 34, 45, 47, 59 and 78 years) (BioChain Institute Inc., Newark, CA, USA) was used. cDNA was synthesized from $2 \mu \mathrm{g}$ total RNA by reverse transcription using the SuperScript III First Strand cDNA Synthesis system (Thermo Fisher Scientific, K.K., Tokyo, Japan). The cDNA synthesis was primed by random hexamers.

Quantitative polymerase chain reaction ( $q P C R$ ) of wt-HER2 and delta-HER2. The delta-HER2 lacks exon 16, from which 16 amino acids are coded. Exon 16 is positioned at the extracellular domain close to the transmembrane region. The PCR primers used in the present study were designed to specifically amplify each transcript of wt-HER2 and delta-HER2. The primer sequences were as follows: wt-HER2, forward 5'-TCC TGT GTG GAC CTG GAT GA-3'; delta-HER2, forward 5'-GCA CCC ACT CCC CTC TGA-3'; and common reverse primer, 5'-CGC TTG ATG AGG ATC CCA AA-3' (FASMAC Co. Ltd., Kanagawa, Japan) (Fig. 1). qPCR was conducted using SsoFast $^{\mathrm{TM}}$ EvaGreen ${ }^{\circledR}$ Supermix with Low ROX (Bio-Rad Laboratories, Inc., Tokyo, Japan). A $20 \mu \mathrm{l}$ reaction solution contained $300 \mathrm{nM}$ forward and reverse primers and cDNA transcribed from $40 \mathrm{ng}$ total RNA as a template. The reaction program was initiated by denaturation at $96^{\circ} \mathrm{C}$ for $5 \mathrm{~min}$, followed by 40 cycles at $96^{\circ} \mathrm{C}$ for $5 \mathrm{sec}$ and $67^{\circ} \mathrm{C}$ for $30 \mathrm{sec}$. The quantification of 18S rRNA was conducted using TaqMan Master Mix and 18S rRNA Primer and Probe kit (Thermo Fisher Scientific, K.K.) with cDNA transcribed from 40 ng total
RNA. The reaction program was initiated by $50^{\circ} \mathrm{C}$ for $2 \mathrm{~min}$ and $95^{\circ} \mathrm{C}$ for $10 \mathrm{~min}$, followed by 40 cycles at $95^{\circ} \mathrm{C}$ for $15 \mathrm{sec}$ and $60^{\circ} \mathrm{C}$ for $1 \mathrm{~min}$. The changes in fluorescence were monitored using the ABI Prism 7000 Sequence Detection system (Thermo Fisher Scientific, K.K.).

Quantification cycle $(\mathrm{Cq})$ of wt-HER2 $\left(\mathrm{Cq}_{\mathrm{wt}-\mathrm{HER} 2}\right)$, delta-HER2 $\left(\mathrm{Cq}_{\text {delta-HER2 }}\right)$ and $18 \mathrm{~S}$ rRNA $\left(\mathrm{Cq}_{18 \mathrm{~S} \text { rRNA }}\right)$ was determined as the cycle where the linear increase in fluorescence reached the threshold level. Firstly, wt-HER2 and delta-HER2 were standardized by $18 \mathrm{~S}$ rRNA, and $\Delta \mathrm{Cq}$ was calculated as $\Delta \mathrm{Cq}_{\mathrm{wt}-\mathrm{HER} 2}=\mathrm{Cq}_{\mathrm{wt}-\mathrm{HER} 2}-\mathrm{Cq}_{18 \mathrm{~S} \text { rRNA }}$ and $\Delta \mathrm{Cq}_{\text {delta-HER2}}=\mathrm{Cq}_{\text {delta-HER }}$ ${ }_{2}-\mathrm{Cq}_{18 \mathrm{~S} \text { rRNA }}$, respectively. The relative expression levels were then calculated using the $2^{-\Delta \Delta \mathrm{Cq}}$ formula (17), where $\Delta \Delta \mathrm{Cq}$ was calculated by subtracting $\Delta \mathrm{Cq}$ of normal breast tissues from $\Delta \mathrm{Cq}$ of breast cancer tissues. The mRNA expression levels of wt-HER 2 and delta-HER 2 in breast cancer tissues were calculated as a fold increase or decrease relative to normal breast tissues.

The specificity of PCR was determined by the amplification of wt-HER 2 and delta-HER2 from the mixture of wt-HER2 and delta-HER2 templates. The templates were mixtures of purified PCR products of wt-HER2 and delta-HER2 at concentration ratios of 100:0.1, 10:1, 1:10, 0.1:100 fg/tube, respectively. PCR was conducted by the same method used for qPCR. After the reaction, the PCR products were electrophoresed on $3 \%$ agarose gel.

Immunostaining and scoring. Paraffin-embedded sections (4 $\mu \mathrm{m})$ were immunostained with antibodies against estrogen receptor (ER; clone SP1; cat. no. 790-4324; $100 \mu 1$ of prediluted antibody; Roche Diagnostics K.K., Tokyo, Japan), progesterone receptor (PgR; clone 1E2; cat. no. 790-2223; $100 \mu \mathrm{l}$ of prediluted antibody; Roche Diagnostics K.K.), Ki-67 (clone MIB-1; cat. no. M7240; 1:100 dilution; DAKO Japan, Tokyo, Japan) and activated form of phosphorylated SRC (pSRC; Tyr416; cat. no. 2101; 1:100 dilution; Cell Signaling Technology Japan, K.K., Tokyo, Japan). Immunostaining was conducted using the Ventana BenchMark GX (Roche Diagnostics K.K.) automated staining system, and the peroxidase reaction was visualized by diaminobenzidine.

Nuclear staining was considered positive for ER, PgR and Ki-67. The expression of ER and PgR was scored according to the method reported by Allred et al (18), and a total score $\geq 3$ was considered positive. The Ki-67 index was calculated as a ratio (\%) of the number of positive nuclei per 1,000 neoplastic cells in the area of the highest labeling, which is also known as the 'hot spot.' Cytoplasmic staining close to the cell membrane was considered positive for $\mathrm{pSRC}$, and the expression was scored semiquantitatively as follows. When there was no staining, the tumor was scored as 0 . If the cells were stained, the staining was scored between 1 and 3 . A score of 1 was defined as weak staining, the same as some normal epithelial cells; a score of 3 was defined as strong membranous and cytoplasmic staining. A score of 2 indicated staining intensity between scores 1 and 3 .

Hierarchical clustering and statistical analysis. Hierarchical clustering and statistical analysis were performed using JMP 10 (SAS Institute Japan, Tokyo, Japan). The comparison between two groups was conducted using Mann-Whitney U 
Table I. Clinicopathological features of patients with HER2-overexpressing breast cancer.

\begin{tabular}{|c|c|c|c|c|c|c|c|c|c|c|}
\hline No. & $\begin{array}{l}\text { Age } \\
(\mathrm{yr})\end{array}$ & $\begin{array}{l}\text { Intrinsic } \\
\text { subtype }\end{array}$ & $\mathrm{pT}$ & $\begin{array}{c}\text { Ki-67 } \\
(\%)\end{array}$ & $\mathrm{LN}$ & Metastasis & $\begin{array}{l}\text { wt-HER2 } \\
\text { (fold) }\end{array}$ & $\begin{array}{l}\text { delta-HER2 } \\
\text { (fold) }\end{array}$ & $\begin{array}{c}\text { delta/wt } \\
(\%)\end{array}$ & pSRC \\
\hline 1 & 35 & LumB & 2 & 49.6 & - & & 46.2 & 32.3 & 1.5 & 2 \\
\hline 2 & 38 & LumB & 3 & 19.0 & + & $\mathrm{Br}$ & 54.8 & 49.5 & 3.9 & 1 \\
\hline 3 & 42 & HER $2^{+}$ & 2 & 82.0 & + & Ms & 16.6 & 25.7 & 3.4 & 1 \\
\hline 4 & 42 & LumB & 1 & 16.7 & - & $\mathrm{Br}, \mathrm{Li}$ & 4.0 & 3.1 & 1.7 & 1 \\
\hline 5 & 42 & LumB & 2 & 38.5 & + & $\mathrm{Br}$ & 1.1 & 1.1 & 2.1 & 0 \\
\hline 6 & 42 & LumB & 1 & 69.3 & + & & 23.3 & 46.4 & 3.4 & 0 \\
\hline 7 & 43 & ${\text { HER } 2^{+}}^{+}$ & 1 & 52.7 & + & & 3.6 & 5.4 & 3.4 & 2 \\
\hline 8 & 43 & HER $2^{+}$ & - & 87.5 & + & Li, Lg, Bn, LN & 41.5 & 50.9 & 2.1 & 3 \\
\hline 9 & 43 & LumB & 1 & 42.1 & - & & 10.2 & 8.7 & 1.9 & 3 \\
\hline 10 & 45 & HER $2^{+}$ & 1 & 44.3 & + & $\mathrm{Br}$ & 7.5 & 21.0 & 6.2 & 1 \\
\hline 11 & 46 & LumB & 2 & 55.5 & + & $\mathrm{Br}$, Skin & 5.7 & 7.2 & 2.8 & 0 \\
\hline 12 & 47 & LumB & 1 & 29.4 & + & & 0.5 & 0.3 & 1.4 & 1 \\
\hline 13 & 48 & HER $2 ~^{+}$ & 1 & 44.9 & - & $\mathrm{Br}$ & 6.6 & 8.1 & 2.7 & 1 \\
\hline 14 & 48 & LumB & 1 & 79.4 & - & & 94.4 & 222.1 & 3.0 & 2 \\
\hline 15 & 49 & LumB & 2 & 44.5 & - & $\mathrm{Br}, \mathrm{Bn}$ & 598.4 & 19.3 & 0.1 & 2 \\
\hline 16 & 50 & LumB & 2 & 75.2 & - & & 169.5 & 150.1 & 2.0 & 3 \\
\hline 17 & 50 & LumB & 4 & 43.7 & + & & 25.4 & 15.7 & 1.5 & 2 \\
\hline 18 & 50 & LumB & 2 & 69.4 & - & & 39.7 & 40.4 & 1.8 & 3 \\
\hline 19 & 51 & ${\text { HER } 2^{+}}^{+}$ & 2 & 74.8 & + & & 113.4 & 83.6 & 1.3 & 3 \\
\hline 20 & 52 & LumB & 1 & 58.4 & - & & 4.1 & 4.0 & 2.2 & 3 \\
\hline 21 & 53 & HER $2^{+}$ & 1 & 78.2 & - & & 24.9 & 20.6 & 1.9 & 3 \\
\hline 22 & 55 & HER $2^{+}$ & 2 & 63.4 & - & & 177.3 & 156.0 & 1.5 & 0 \\
\hline 23 & 56 & HER $2^{+}$ & 4 & 69.7 & + & & 432 & 499.7 & 2.6 & 2 \\
\hline 24 & 57 & HER $2^{+}$ & 2 & 92.3 & + & & 87.7 & 114.2 & 2.3 & 2 \\
\hline 25 & 57 & LumB & 1 & 67.3 & - & & 0.8 & 0.6 & 1.6 & 2 \\
\hline 26 & 59 & ${\text { HER } 2^{+}}^{+}$ & 1 & 64.7 & + & & 9.3 & 7.8 & 1.9 & 2 \\
\hline 27 & 60 & ${\text { HER } 2^{+}}^{+}$ & 1 & 68.5 & - & & 70.0 & 56.1 & 1.4 & 3 \\
\hline 28 & 61 & HER $2^{+}$ & 1 & 51.2 & - & & 2.0 & 1.8 & 2.1 & 1 \\
\hline 29 & 64 & HER $2^{+}$ & 2 & 68.2 & + & & 6.7 & 3.8 & 1.3 & 1 \\
\hline 30 & 64 & HER $2^{+}$ & 1 & 43.7 & + & & 1.7 & 2.4 & 3.2 & 0 \\
\hline 31 & 64 & HER $2+^{+}$ & 1 & 31.8 & - & & 100.8 & 177.3 & 3.0 & 1 \\
\hline 32 & 65 & LumB & 1 & 56.4 & - & & 315.2 & 128.9 & 0.7 & 3 \\
\hline 33 & 68 & ${\text { HER } 2^{+}}^{+}$ & 1 & 88.0 & + & & 15.1 & 20.2 & 2.3 & 1 \\
\hline 34 & 69 & ${\text { HER } 2^{+}}^{+}$ & 4 & 92.3 & + & $\mathrm{LN}$ & 30.6 & 32.9 & 2.4 & 3 \\
\hline 35 & 72 & HER $2^{+}$ & 2 & 72.0 & - & & 4.1 & 3.9 & 1.7 & 0 \\
\hline 36 & 73 & LumB & 1 & 28.5 & + & & 10.9 & 9.8 & 2.0 & 3 \\
\hline 37 & 73 & LumB & 1 & 52.7 & - & & 11.8 & 6.7 & 1.0 & 3 \\
\hline 38 & 78 & LumB & 2 & 74.4 & + & & 55.7 & 64.9 & 2.0 & 3 \\
\hline 39 & 81 & ${\text { HER } 2^{+}}^{+}$ & 2 & 34.6 & + & & 3.9 & 3.6 & 2.1 & 3 \\
\hline 40 & 85 & LumB & 2 & 80.1 & - & & 203.7 & 370.9 & 2.4 & 3 \\
\hline
\end{tabular}

Bn, bone; Br, brain; HER2 ${ }^{+}$, human epidermal growth factor receptor 2-positive; LumB, luminal B; Lg, lung; Li, liver; LN, lymph node; Ms, muscle; pSRC, phosphorylated-SRC; pT, primary tumor staging; wt, wild type; yr, years of the age.

test, and the comparison among three groups was performed using the Steel-Dwass method. The regressions between wt-HER2 and delta-HER2 mRNA levels and between wt-HER2 mRNA level and delta/wt ratio was examined by regression analysis. $\mathrm{P}<0.05$ was considered to indicate a statistically significant difference.

\section{Results}

Clinicopathological features of HER2-overexpressing breast cancer. A total of 40 cases of HER2-overexpressing breast cancer were studied in the present study, all of which were women, aged between 35 and 85 years (Table I). Breast 


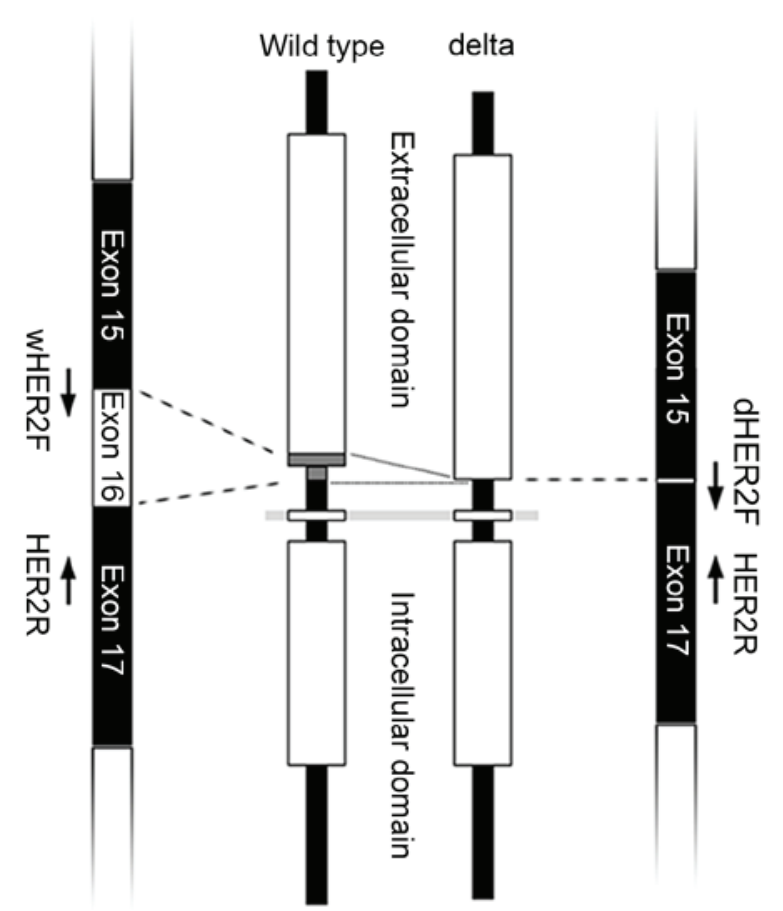

Figure 1. Structure of wt-HER2 and delta-HER2, and PCR primers for mRNA expression analysis.

cancer histology (Fig. 2) observed sheet-like, cribriform and trabecular proliferation of atypical cells, which possessed irregular-shaped nuclei with prominent nucleoli and frequent mitotic figures (Fig. 2A). All cases were diagnosed as invasive carcinoma and histological grades were high. Strong expression of HER 2 was identified on the cell membrane (Fig. 2B). A total of 20 cases were classified as hormone receptor-positive luminal B subtype (Fig. 2C), and the other 20 cases were classified as hormone receptor-negative HER2-positive subtype. The Ki-67 index ranged between 19.0 and 92.3\% (Fig. 2D). A total of 23 cases had lymph node metastasis at the time of operation, and recurrence was noted in 10 cases.

mRNA expression levels of wt-HER2 and delta-HER2. The specificity of qPCR was confirmed by amplifying a mixture of sequentially diluted wt-HER 2 and delta-HER 2 mRNA (Fig. 3A). The amount of each amplified product correlated with the amount of target in the mixture, and no cross-reaction was detected.

The expression levels of wt-HER2 and delta-HER2 varied largely, from 0.5 to 598.4 -fold and from 0.3 to 499.7 -fold compared with normal breast tissues, respectively (Table I). There was a power regression between wt-HER2 and delta-HER2 mRNA levels $\left(r^{2}=0.86, \mathrm{P}<0.0001\right.$; Fig. 3B). The delta/wt ratio ranged between 0.1 and $6.2 \%$ (Fig. $3 \mathrm{C}$ ); the average was $2.2 \pm 1.0 \%$. There was a weak inverse correlation between delta/wt ratio and the mRNA expression levels of wt-HER2 ( ${ }^{2}=0.1$, Fig. 3C).

Immunostaining of $p S R C$. Immunostaining of the activated form of pSRC was negative in six cases (15\%). Weak and moderate positive immunostaining, scored as 1 and 2, was detected in 10 and nine cases, respectively (25 and 23\%)
Table II. Comparison of clinicopathological features among the human epidermal growth factor receptor 2-overexpressing breast cancer clusters.

\begin{tabular}{lcccc}
\hline & \multicolumn{3}{c}{ Clusters } & \\
\cline { 2 - 4 } Variable & 1 & 2 & 3 & P-value \\
\hline Intrinsic subtype & & & & $\mathrm{P}=0.92$ \\
Luminal B & 9 & 6 & 5 & \\
HER2-positive & 10 & 6 & 4 & \\
pT & & & & $\mathrm{P}=0.753$ \\
1 & 11 & 6 & 3 & \\
2 & 6 & 4 & 5 & \\
3 & 1 & 0 & 0 & \\
4 & 1 & 1 & 1 & \\
Lymph node status & & & & $\mathrm{P}=0.065$ \\
- & 6 & 6 & 7 & \\
+ & 13 & 6 & 2 & \\
Recurrence & & & & $\mathrm{P}=0.236$ \\
- & 12 & 10 & 8 & \\
+ & 7 & 2 & 1 & \\
\hline
\end{tabular}

pT, primary tumor staging.

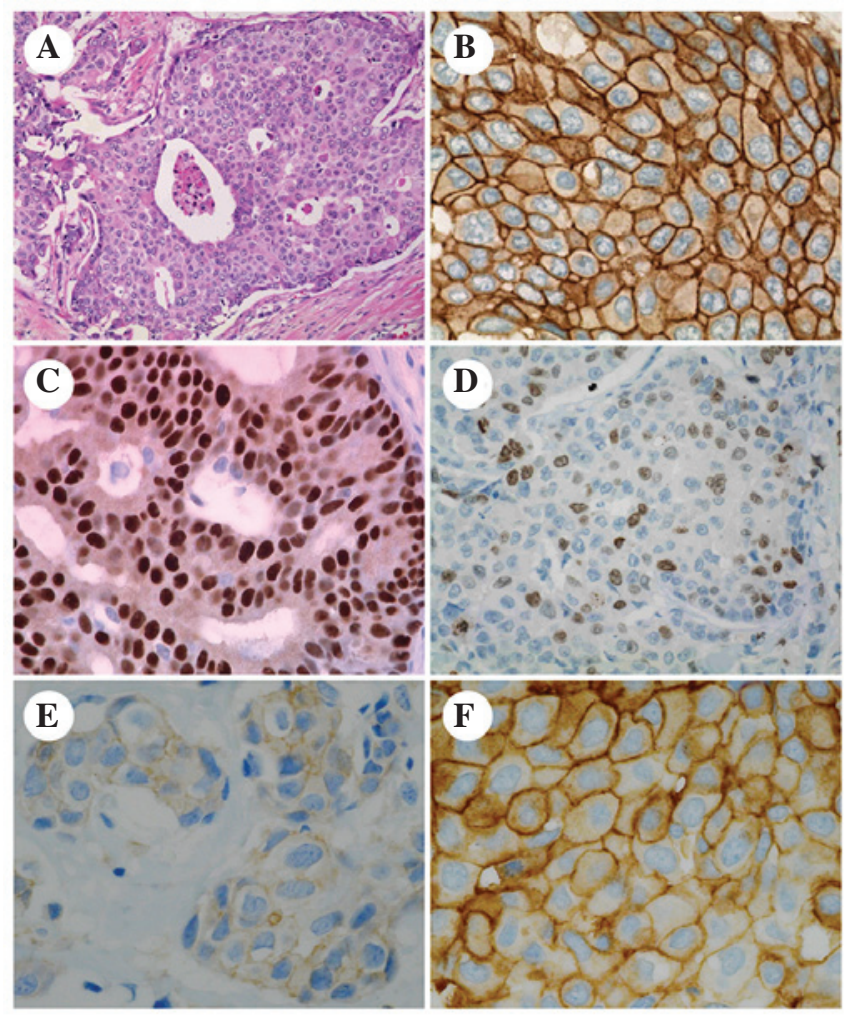

Figure 2. Histological features of HER2-positive breast cancer. (A) Sheet-like proliferation of carcinoma cells, which possessed irregular-shaped nuclei with prominent nucleoli (x40 magnification). (B) Strong continuous expression of HER2 was observed on the cell membrane (x200 magnification). (C) Positive reaction of estrogen receptor in tumor cells (x200 magnification). (D) Representative immunostaining of Ki-67 in tumor tissue (x200 magnification). Representative immunostaining of phosphorylated-SRC of (E) score 1 and (F) score 3 (x400 magnification). HER2, human epidermal growth factor receptor 2 . 


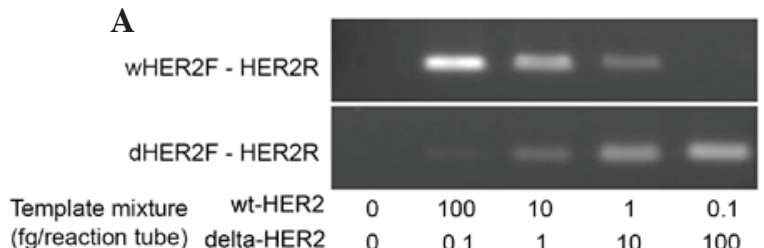

B

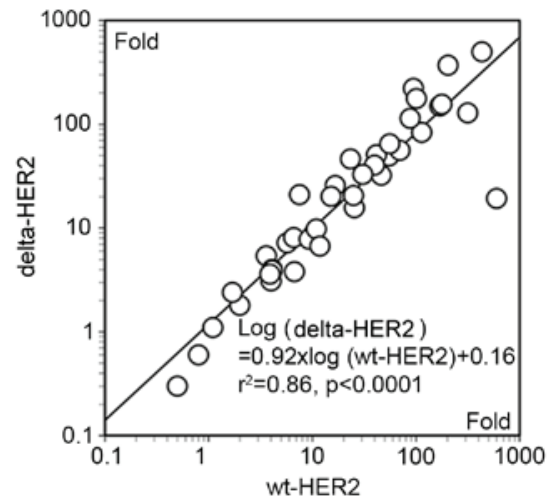

C

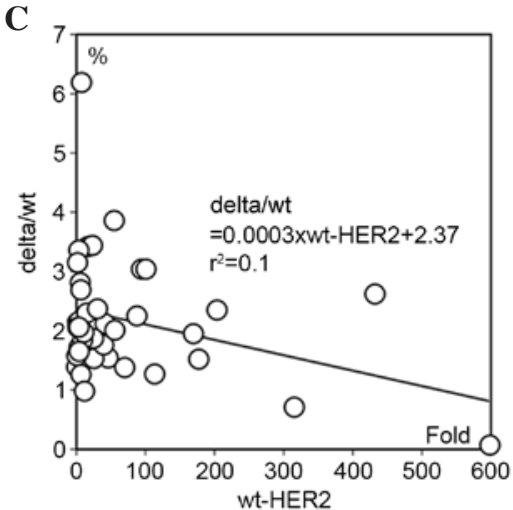

Figure 3. Molecular features and mRNA expression levels of wt-HER2 and delta-HER2. (A) Electrophoresis of PCR products amplified from a mixture of wt-HER2 and delta-HER2 templates. (B) Correlation between wt-HER2 and delta-HER2 mRNA expression levels. (C) Correlation between wt-HER2 and delta/wt ratio. The ratio tends to be slightly high in the cases with low mRNA expression levels of delta-HER2. HER2, human epidermal growth factor receptor 2; PCR, polymerase chain reaction; wt, wild type.

(Fig. 2E). Strong immunostaining of pSRC, scored as 3, was noted in 15 cases (38\%; Fig. 2F). The strong positive reaction was detected on the cell membrane and in the cytoplasm close to the cell membrane.

Hierarchical clustering and clinicopathological features. Hierarchical clustering with wt-HER2, delta-HER2 and pSRC separated the HER2-overexpressing breast cancer cases into three major clusters (Fig. 4A). In cluster 1, the expression levels of wt-HER2 and delta-HER2 were lower than those in the other clusters (Fig. 4B and C). The mRNA expression levels of wt-HER2 and delta-HER2 were elevated in clusters 2 and 3, and the pSRC score was high in the majority of cases in these clusters (Fig. 4A-C). Delta/wt ratio appeared to be slightly higher in cluster 1 compared with those in the other clusters; however, the difference did not reach statistical significance (Fig. 4D). The Ki-67 index was high in all clusters; however, a trend was observed that the index was lower in cluster 1 compared with in clusters 2 and 3 (Fig. 4E).

The clinicopathological characteristics of cases in the three clusters were analyzed (Table II). There was no difference in the intrinsic subtypes and primary tumor staging among the clusters. Lymph node status and recurrence were more frequent in cluster 1; however, the differences did not reach statistical significance (Table II).

Association between delta/wt ratio and clinicopathological features. Associations between the delta/wt ratio and clinicopathological features, such as intrinsic subtypes, lymph node status and disease recurrence were further analyzed. There was no significant difference in delta/wt ratio between HER2-positive and luminal B cases (Fig. 5A). However, the $\mathrm{delta} / \mathrm{wt}$ ratio was significantly elevated in lymph node-positive cases (Fig. 5B) and in cases with recurrence (Fig. 5C). No significant differences in wt-HER 2 and delta-HER2 mRNA levels were detected between intrinsic subtypes, lymph node status and recurrence (data not shown).

\section{Discussion}

The present study detected delta-HER 2 mRNA expression, a splice variant of HER2, in Japanese patients with HER2-overexpressing breast cancer. The mRNA expression levels of delta-HER2 mRNA appeared to be correlated with those of wt-HER2 mRNA. The HER2-overexpressing breast cancer were separated into cancer with elevated delta-HER2 and enhanced pSRC, and cancer with low expression level of delta-HER2, but with elevated delta/wt ratio. Furthermore, the delta/wt ratio was significantly increased in breast cancer cases with lymph node metastasis and recurrence.

In the present study, the breast cancer cases were separated into three clusters by hierarchical clustering with wt-HER2, delta-HER 2 and pSRC. Tumors with moderate and high mRNA expression levels of wt-HER2 and delta-HER2 (clusters 2 and 3; Fig. 4A) presented enhanced phosphorylation of SRC and an increased Ki-67 index. These results are consistent with the findings of a previous report, which indicated that overexpression of delta-HER2 induces phosphorylation of SRC and stimulates the proliferative activity of breast cancer cells (9). It is also plausible that the expression of other HER family molecules, such as epidermal growth factor receptor and HER3, may affect the phosphorylation of SRC; however, the expression of these molecules was not examined in the present study $(11,19)$. It is conceivable that HER2-overexpressing breast cancer cases are heterogeneous in the expression of wt-HER2 and delta-HER2, and intracellular signaling.

In 19 out of 40 cases (48\%), delta- and wt-HER 2 expression was relatively low, and SRC was only moderately phosphorylated or remained unphosphorylated (cluster 1; Fig. 4A). In addition, in cluster 1 the Ki-67 index appeared slightly lower 
A

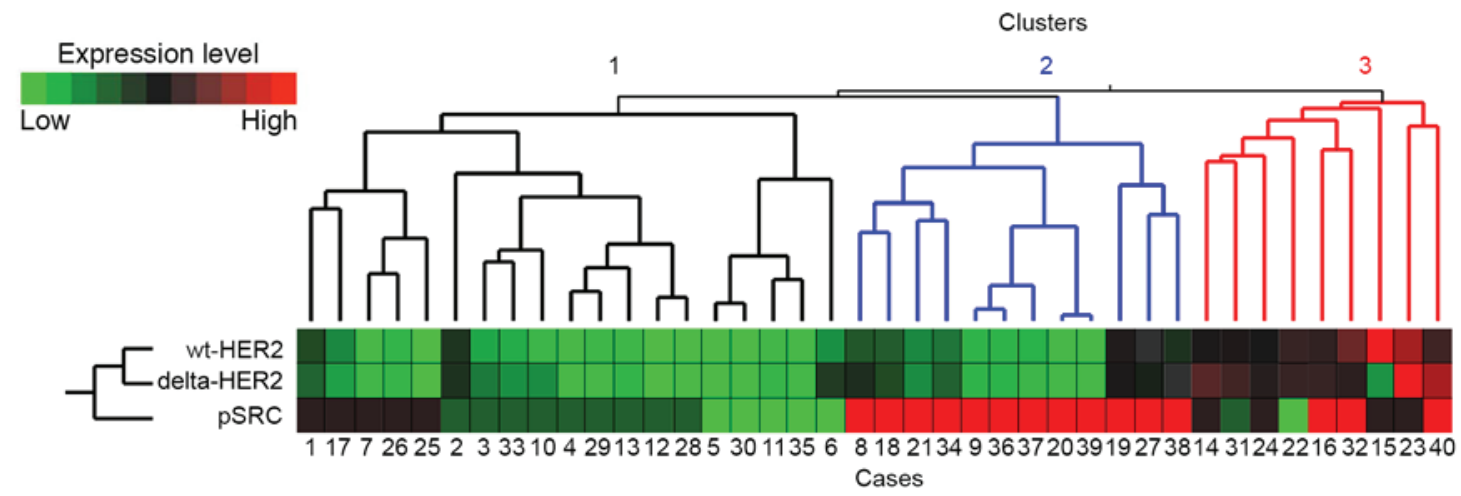

B

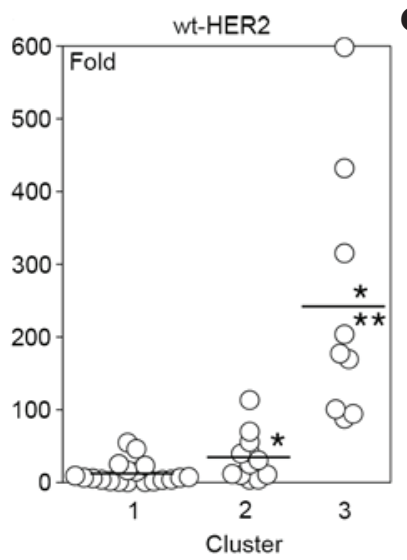

C

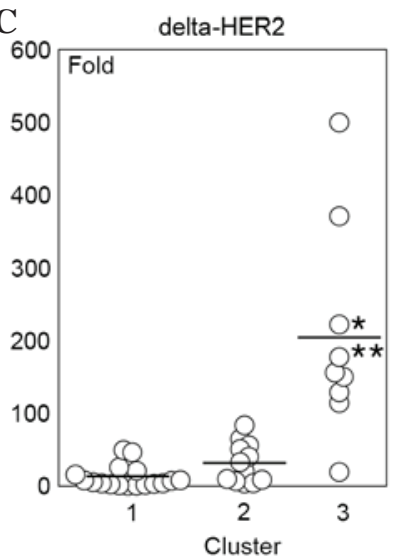

D

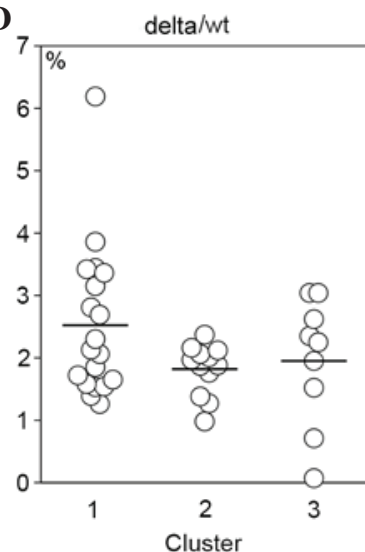

E

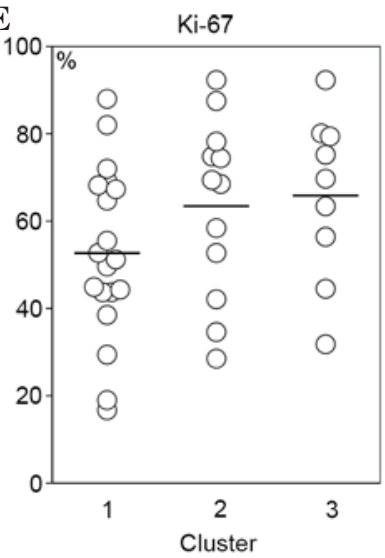

Figure 4. Hierarchical clustering of wt-HER2, delta-HER2 and pSRC, and association with clinicopathological factors. (A) Unsupervised hierarchical clustering of wt-HER2 and delta-HER2 and pSRC separated the breast cancer cases into three major clusters. The levels of (B) wt-HER2 mRNA and (C) delta-HER2 mRNA were significantly higher in clusters 2 and $3\left({ }^{*} \mathrm{P}<0.05\right.$ vs. cluster $1,{ }^{* *} \mathrm{P}<0.05$ vs. cluster 2$)$. (D) The delta/wt ratio tended to be higher and (E) Ki-67 index tended to be lower in cluster 1, compared with in clusters 2 and 3. HER2, human epidermal growth factor receptor 2; pSRC, phosphorylated-SRC; wt, wild type.
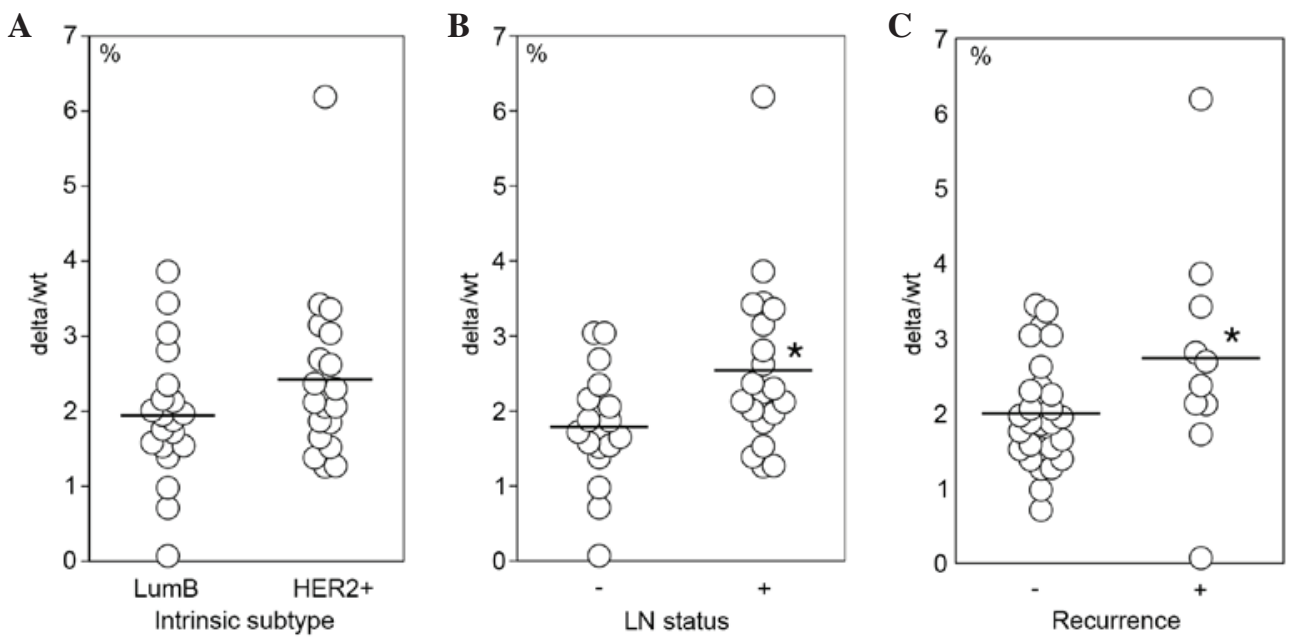

Figure 5. Association between the delta/wt human epidermal growth factor receptor 2 ratio and clinicopathological features. (A) Delta/wt ratio was comparable between intrinsic subtypes. Delta/wt ratio was significantly higher in (B) LN-positive cases ( $\mathrm{P}<0.05$ vs. LN-negative cases) and in (C) cases with recurrence $\left({ }^{*} \mathrm{P}<0.05\right.$ vs. cases without recurrence). LN, lymph node.

compared with in the other clusters (Fig. 4D). However, there was a trend that lymph node metastasis was more frequent in these patients compared with the patients from the other clusters (Table II). This finding appears to be in contrast with that of a previous report, which demonstrated that high frequency of lymph node metastasis was present in cases with high expression levels of delta-HER2 (9). Furthermore, cases with a 'high delta-HER2 signature', which was identified in a cell line with delta-HER2 overexpression, had a worse prognosis in distant metastasis-free survival (12). In the current 
study, the follow-up duration may be too short to determine effects on worse prognostic factors, such as local recurrence and distant metastasis. In addition, the number of patients in the present study was limited. There may also be ethnic differences in the biological behavior of HER2-overexpressing breast cancer (20).

The delta/wt ratio in cluster 1 exhibited a trend to be higher than in the other clusters. Therefore, the association between clinicopathological characteristics and the delta/wt ratio was further examined. The delta/wt ratio was significantly higher in cases with lymph node metastasis and recurrence compared with in the cases without them. The mRNA expression levels of delta-HER2 and wt-HER2 were comparable in the two groups. Delta/wt ratio may be a good biological marker for the prediction of aggressive behavior of HER2-overexpressing breast cancer in Japanese patients. It may be valuable to use formalin-fixed paraffin-embedded specimens for the quantification of wt-HER2 and delta-HER2 mRNA expression.

The efficacy of trastuzumab on delta-HER2-overexpressing breast cancer is controversial. Delta-HER2 may chemically dimerize through disulfide bond formation and stimulate the signaling pathway to promote proliferation of tumor cells $(8,11,13)$. In a previous study, proliferation was not inhibited by trastuzumab in tumor cells overexpressing delta-HER2 in vitro $(8,9)$. Conversely, in vivo, the growth of implanted tumor cells in nude mice was inhibited by trastuzumab $(12,14)$. Furthermore, human patients with high levels of delta-HER2 and enhanced pSRC followed a better clinical course, as compared with the cases with low levels of delta-HER2 and pSRC, following treatment with trastuzumab (14). The discrepancy of the efficacy of trastuzumab between in vitro and in vivo conditions may, in part, be due to the presence of immunological machinery against HER2-overexpressing tumor cells in vivo (21). Further examination is required to elucidate the effects of trastuzumab on Japanese patients with HER2-overexpressing breast cancer.

In conclusion, the current study demonstrated the expression of delta-HER2 in HER2-overexpressing breast cancer from Japanese patients. The enhanced expression of delta-HER 2 was associated with phosphorylation of SRC, whereas the increase in the delta/wt ratio was associated with lymph node metastasis and recurrence. The delta/wt ratio may be a valuable biomarker to predict the prognosis of HER2-overexpressing breast cancer. Further examination is required to elucidate the precise molecular effects induced by delta-HER2.

\section{Acknowledgements}

The authors of the present study would like to thank Dr Shunji Aizawa and Dr Akimasa Nishimura for their assistance and invaluable suggestions.

\section{References}

1. Barnard ME, Boeke CE and Tamimi RM: Established breast cancer risk factors and risk of intrinsic tumor subtypes. Biochim Biophys Acta 1856: 73-85, 2015.

2. Perou CM, Sørlie T, Eisen MB, van de Rijn M, Jeffrey SS, Rees CA, Pollack JR, Ross DT, Johnsen H, Akslen LA, et al: Molecular portraits of human breast tumours. Nature 406 : $747-752,2000$
3. Slamon DJ, Clark GM, Wong SG, Levin WJ, Ullrich A and McGuire WL: Human breast cancer: Correlation of relapse and survival with amplification of the HER-2/neu oncogene. Science 235: 177-182, 1987.

4. Sørlie T, Perou CM, Tibshirani R, Aas T, Geisler S, Johnsen H, Hastie T, Eisen MB, van de Rijn M, Jeffrey SS, et al: Gene expression patterns of breast carcinomas distinguish tumor subclasses with clinical implications. Proc Natl Acad Sci USA 98: 10869-10874, 2001.

5. Lobbezoo DJ, van Kampen RJ, Voogd AC, Dercksen MW, van den Berkmortel F, Smilde TJ, van de Wouw AJ, Peters FP, van Riel JM, Peters NA, et al: Prognosis of metastatic breast cancer subtypes: The hormone receptor/HER2-positive subtype is associated with the most favorable outcome. Breast Cancer Res Treat 141: 507-514, 2013.

6. Scott GK, Robles R, Park JW, Montgomery PA, Daniel J, Holmes WE, Lee J, Keller GA, Li WL, Fendly BM, et al: A truncated intracellular HER2/neu receptor produced by alternative RNA processing affects growth of human carcinoma cells. Mol Cell Biol 13: 2247-2257, 1993.

7. Jackson C, Browell D, Gautrey $\mathrm{H}$ and Tyson-Capper A: Clinical significance of HER-2 splice variants in breast cancer progression and drug resistance. Int J Cell Biol 2013: 973584 , 2013.

8. Castiglioni F, Tagliabue E, Campiglio M, Pupa SM, Balsari A and Ménard S: Role of exon-16-deleted HER2 in breast carcinomas. Endocr Relat Cancer 13: 221-232, 2006.

9. Mitra D, Brumlik MJ, Okamgba SU, Zhu Y, Duplessis TT, Parvani JG, Lesko SM, Brogi E and Jones FE: An oncogenic isoform of HER 2 associated with locally disseminated breast cancer and trastuzumab resistance. Mol Cancer Ther 8: 2152-2162, 2009.

10. Kwong KY and Hung MC: A novel splice variant of HER2 with increased transformation activity. Mol Carcinog 23: 62-68, 1998.

11. Siegel PM, Ryan ED, Cardiff RD and Muller WJ: Elevated expression of activated forms of Neu/ErbB-2 and ErbB-3 are involved in the induction of mammary tumors in transgenic mice: Implications for human breast cancer. EMBO J 18: 2149-2164, 1999.

12. Alajati A, Sausgruber N, Aceto N, Duss S, Sarret S, Voshol H, Bonenfant D and Bentires-Alj M: Mammary tumor formation and metastasis evoked by a HER2 splice variant. Cancer Res 73: 5320-5327, 2013

13. Marchini C, Gabrielli F, Iezzi M, Zenobi S, Montani M, Pietrella L, Kalogris C, Rossini A, Ciravolo V, Castagnoli L, et al: The human splice variant $\triangle 16$ HER 2 induces rapid tumor onset in a reporter transgenic mouse. PLoS One 6: e18727, 2011.

14. Castagnoli L, Iezzi M, Ghedini GC, Ciravolo V, Marzano G, Lamolinara A, Zappasodi R, Gasparini P, Campiglio M, Amici A, et al: Activated d16HER2 homodimers and SRC kinase mediate optimal efficacy for trastuzumab. Cancer Res 74: 6248-6259, 2014

15. Lakhani SR, Ellis IO, Schnitt SJ, Tan PH and van de Vijver MJ: WHO Classification of tumours of the breast. IARC, Lyon, 2012.

16. Wolff AC, Hammond ME, Hicks DG, Dowsett $M$, McShane LM, Allison KH, Allred DC, Bartlett JM, Bilous M, Fitzgibbons P, et al: Recommendations for human epidermal growth factor receptor 2 testing in breast cancer: American society of clinical oncology/college of American pathologists clinical practice guideline update. J Clin Oncol 31: 3997-4013, 2013.

17. Livak KJ and Schmittgen TD: Analysis of relative gene expression data using real-time quantitative PCR and the 2(-Delta Delta C(T)) Method. Methods 25: 402-408, 2001.

18. Allred DC, Harvey JM, Berardo M and Clark GM: Prognostic and predictive factors in breast cancer by immunohistochemical analysis. Mod Pathol 11: 155-168, 1998.

19. Zhang S, Huang WC, Li P, Guo H, Poh SB, Brady SW, Xiong Y, Tseng LM, Li SH, Ding Z, et al: Combating trastuzumab resistance by targeting SRC, a common node downstream of multiple resistance pathways. Nat Med 17: 461-469, 2011.

20. Natori A, Hayashi N, Soejima K, Deshpande GA, Takahashi O, Cristofanilli M, Ueno NT and Yamauchi H: A comparison of epidemiology, biology, and prognosis of inflammatory breast cancer in Japanese and US populations. Clin Breast Cancer 13: 460-464, 2013

21. Bianchini G and Gianni L: The immune system and response to HER2-targeted treatment in breast cancer. Lancet Oncol 15: e58-e68, 2014. 